PROCEEDINGS OF THE

AMERICAN MATHEMATICAL SOCIETY

Volume 137, Number 11, November 2009, Pages 3647-3656

S 0002-9939(09)09951-1

Article electronically published on June 12, 2009

\title{
TROPICALIZATION AND IRREDUCIBILITY OF GENERALIZED VANDERMONDE DETERMINANTS
}

\author{
CARLOS D'ANDREA AND LUIS FELIPE TABERA
}

(Communicated by Martin Lorenz)

\begin{abstract}
We find geometric and arithmetic conditions in order to characterize the irreducibility of the determinant of the generic Vandermonde matrix over the algebraic closure of any field $k$. We also characterize those determinants whose tropicalization with respect to the variables of a row is irreducible.
\end{abstract}

\section{INTRODUCTION}

Let $n, N$ be positive integers, let $\mathbf{X}_{1}, \ldots, \mathbf{X}_{N}$ be $n$-tuples of indeterminates, i.e.

$$
\mathbf{X}_{i}:=\left(X_{i 1}, \ldots, X_{i n}\right), \quad i=1, \ldots, N,
$$

where each $X_{i j}$ is an indeterminate, and let $\Gamma:=\left(\gamma_{1}, \ldots, \gamma_{N}\right)$ be an $N$-tuple of vectors in $\mathbb{N}^{n}$, with $\gamma_{j}=\left(\gamma_{j 1}, \ldots, \gamma_{j n}\right)$. Set

$$
V(\underline{\mathbf{X}}, \Gamma):=\left|\begin{array}{ccc}
X_{11}^{\gamma_{11}} X_{12}^{\gamma_{12}} \cdots X_{1 n}^{\gamma_{1 n}} & \ldots \ldots & X_{11}^{\gamma_{N 1}} X_{12}^{\gamma_{N 2}} \cdots X_{1 n}^{\gamma_{N n}} \\
X_{21}^{\gamma_{11}} X_{22}^{\gamma_{12}} \cdots X_{2 n}^{\gamma_{1 n}} & \ldots \ldots & X_{21}^{\gamma_{N 1}} X_{22}^{\gamma_{N 2}} \cdots X_{2 n}^{\gamma_{N n}} \\
& \ldots \ldots \ldots & \\
& \ldots & \\
X_{N 1}^{\gamma_{11}} X_{N 2}^{\gamma_{12}} \cdots X_{N n}^{\gamma_{1 n}} & \ldots \ldots & X_{N 1}^{\gamma_{N 1}} X_{N 2}^{\gamma_{N 2}} \cdots X_{N n}^{\gamma_{N n}}
\end{array}\right| .
$$

We call the polynomial $V(\underline{\mathbf{X}}, \Gamma) \in \mathbb{Z}\left[\left(X_{i, j}\right)_{1 \leq i \leq N, 1 \leq j \leq n}\right]$ the generalized Vandermonde determinant associated to $\Gamma$.

Example 1.1. If $n=1$ and $\Gamma=(0,1, \ldots, N-1)$, then

$$
V(\underline{\mathbf{X}}, \Gamma)= \pm \prod_{1 \leq i<j \leq N}\left(X_{i 1}-X_{j 1}\right)
$$

the classical Vandermonde determinant.

Received by the editors March 14, 2008, and, in revised form, February 27, 2009

2000 Mathematics Subject Classification. Primary 12E05; Secondary 14M12.

Key words and phrases. Vandermonde determinants, multivariate factorization, tropical geometry, valuations.

The first author was supported by the Programa Ramón y Cajal and the Research Project grant MTM2007-67493 from the Ministerio de Educación y Ciencia, Spain.

The second author was supported by the Research Project grant MTM2005-08690-CO2-02 and a postdoctoral fellowship from the Ministerio de Educación y Ciencia, Spain.

(C)2009 American Mathematical Society Reverts to public domain 28 years from publication 
Example 1.2. It is a classical result (see for instance [Mac] that if $n=1$, then for any set $\Gamma \subset \mathbb{N}$ of $N$ elements, the determinant $V(\underline{\mathbf{X}}, \Gamma)$ is a multiple of the classical Vandermonde determinant $\prod_{1 \leq i<j \leq N}\left(X_{i 1}-X_{j 1}\right)$.

Example 1.3. Suppose $n=2, N=3$ and $\Gamma:=((2,0),(0,2),(2,2))$.

By computing the $3 \times 3$ determinant we have that $V(\underline{\mathbf{X}}, \Gamma)$ is equal to

$$
\begin{aligned}
& X_{11}^{2} X_{22}^{2} X_{31}^{2} X_{32}^{2}-X_{11}^{2} X_{32}^{2} X_{21}^{2} X_{22}^{2}-X_{12}^{2} X_{21}^{2} X_{31}^{2} X_{32}^{2} \\
& \quad+X_{12}^{2} X_{31}^{2} X_{21}^{2} X_{22}^{2}+X_{11}^{2} X_{12}^{2} X_{21}^{2} X_{32}^{2}-X_{11}^{2} X_{12}^{2} X_{31}^{2} X_{22}^{2} .
\end{aligned}
$$

Set $\Gamma^{\prime}:=\{(1,0),(0,1),(1,1)\} ;$ it is easy to see that

- If $\operatorname{char}(k) \neq 2, V(\underline{\mathbf{X}}, \Gamma)$ is absolutely irreducible over $k\left[\left(X_{i, j}\right)_{1 \leq i \leq N, 1 \leq j \leq n}\right]$ (i.e. irreducible over $\bar{k}\left[\left(X_{i, j}\right)_{1 \leq i \leq N, 1 \leq j \leq n}\right], \bar{k}$ being the algebraic closure of $k)$.

- If $\operatorname{char}(k)=2$, then $V(\underline{\mathbf{X}}, \Gamma)=V\left(\underline{\mathbf{X}}, \Gamma^{\prime}\right)^{2}$ in $k\left[\left(X_{i, j}\right)_{1 \leq i \leq N, 1 \leq j \leq n}\right]$.

Example 1.4. As an easy exercise, it can be proved that if $\Gamma \subset \mathbb{N}^{n}$ is contained in an affine line, then $V(\underline{\mathbf{X}}, \Gamma)$ factorizes in a similar way as in Example 1.2 .

In the univariate case $(n=1)$, the Vandermonde determinant is associated with the interpolation problem, and it has been extensively studied (see GV, Gow, ElM and the references therein). The multivariate interpolation problem is naturally associated with generalized Vandermonde determinants, and there is also an extensive and current literature on the topic. See for instance [CL, GS, LS, Olv, Zhu].

The purpose of this article is to study the irreducibility of $V(\underline{\mathbf{X}}, \Gamma)$. As Example 1.3 suggests, the answer will depend on the characteristic of $k$. Also, our intuition from the univariate case may lead us to believe that generalized Vandermonde determinants have lots of irreducible factors. Our main result essentially tells us that in general these polynomials are absolutely irreducible.

There are some trivial factors that can already be read from the set of exponents. Let $\bar{\gamma}:=\left(g_{1} \ldots, g_{n}\right)$ where each $g_{i}$ is defined as $\min \left\{\gamma_{1_{i}}, \ldots, \gamma_{N_{i}}\right\}, i=1, \ldots, n$. It is easy to check that the following factorization holds:

$$
V\left(\underline{\mathbf{X}},\left(\gamma_{1}, \ldots, \gamma_{N}\right)\right)=\prod_{i=1}^{n}\left(\prod_{j=1}^{N} X_{i j}\right)^{g_{i}} V\left(\underline{\mathbf{X}},\left(\gamma_{1}-\bar{\gamma}, \ldots, \gamma_{N}-\bar{\gamma}\right)\right),
$$

where $V\left(\underline{\mathbf{X}},\left(\gamma_{1}-\bar{\gamma}, \ldots, \gamma_{N}-\bar{\gamma}\right)\right)$ has no monomial factor. Let $d_{\Gamma}$ be the largest integer such that $\frac{1}{d_{\Gamma}}\left\{\gamma_{1}-\bar{\gamma}, \ldots, \gamma_{N}-\bar{\gamma}\right\} \subset \mathbb{N}^{n}$, and let $\mathcal{L}_{\Gamma} \subset \mathbb{R}^{n}$ be the affine subspace spanned by $\Gamma$.

Theorem 1.5. Let $N \geq 3$. The Vandermonde polynomial $V(\underline{\mathbf{X}}, \Gamma)$ is irreducible in $\bar{k}\left[\left(X_{i j}\right)_{1 \leq i, j, \leq N}\right]$ if and only if the following three conditions apply:

- $\operatorname{dim}\left(\mathcal{L}_{\Gamma}\right) \geq 2$,

- $\operatorname{gcd}\left(\mathbf{X}^{\gamma_{i}}\right)_{1 \leq i \leq N}=1$ or equivalently $\bar{\gamma}=(0, \ldots, 0)$,

- $\operatorname{char}(k)$ does not divide $d_{\Gamma}$.

Note that $\operatorname{dim}\left(\mathcal{L}_{\Gamma}\right) \geq 2$ implies $N \geq 3$ and $n>1$. When $n=2$ and $N=3$ or 4 , Theorem 1.5 can also be obtained from an application of Ostrowski's work [st] on the irreducibility of fewnomials (see also $[\mathrm{BP}]$ ). We will prove the general case by making use of Bertini's theorem on the variety defined by $V(\underline{\mathbf{X}}, \Gamma)$, and applying some results concerning algebraic independence of maximal Vandermonde minors obtained in $\mathrm{Tab}$. 
When dealing with the problem of factorizing multivariate polynomials, several approaches like those given in Ost, Gao focus on the irreducibility of the Newton polytope of the polynomial with respect to the operation of computing Minkowski sums, which gives sufficient conditions to show irreducibility. A refinement of this method can be obtained with the aid of tropical geometry: instead of working with Newton polytopes, we can study regular subdivisions of them. So, we can cover more general families of polynomials, but at the cost of losing track of the characteristic of the ground field $k$.

In our case, the problem can be dealt with as follows: by expanding the determinant of the generalized Vandermonde determinant with respect to the first row, we get the following expansion: $V(\underline{\mathbf{X}}, \Gamma)=\sum_{i=1}^{N}(-1)^{\sigma_{i}} \Delta_{i} \mathbf{X}_{1}^{\gamma_{i}}$. For the irreducibility problem, it is enough to consider a dehomogenized version of $V(\underline{\mathbf{X}}, \Gamma)$ as follows:

$$
V(\underline{\mathbf{X}}, \Gamma)_{\mathbf{a f f}}:=\mathbf{X}_{1}^{\gamma_{N}}+\sum_{i=1}^{N-1} A_{i} \mathbf{X}_{1}^{\gamma_{i}},
$$

where $A_{i}:=(-1)^{\sigma_{i}} \frac{\Delta_{i}}{\Delta_{N}}, i=1, \ldots N-1$.

We can then regard $V(\underline{\mathbf{X}}, \Gamma)$ aff as a polynomial in $K\left[\mathbf{X}_{1}\right], K$ being now a field containing all the $A_{i}$ 's $, i=1, \ldots, N-1$.

Given any rank one valuation $v: K \rightarrow \mathbb{R}$, we can extend it to $K^{n}$ componentwise as follows:

$$
\text { v: } \begin{array}{ccc}
K^{n} & \rightarrow & \mathbb{R}^{n} \\
\left(z_{1}, \ldots, z_{n}\right) & \mapsto & \left(v\left(z_{1}\right), \ldots, v\left(z_{n}\right)\right) .
\end{array}
$$

The tropicalization of $V(\underline{\mathbf{X}}, \Gamma)$ is then defined as

$$
\operatorname{Trop}(V(\underline{\mathbf{X}}, \Gamma))=\overline{\mathbf{v}\left(\left\{V(\underline{\mathbf{X}}, \Gamma)_{\text {aff }}=0\right\}\right)} \subseteq \mathbb{R}^{n},
$$

where the closure on the right hand side is taken with respect to the standard topology in $\mathbb{R}^{n}$.

It turns out (see for instance $[\mathrm{BG}$ ] or $[\mathrm{EKL})$ that $\operatorname{Trop}(V(\underline{\mathbf{X}}, \Gamma))$ is a connected polyhedral complex of codimension 1 . If $V(\underline{\mathbf{X}}, \Gamma)_{\text {aff }}$ is reducible over $K\left[\mathbf{X}_{1}\right]$, then $\operatorname{Trop}(V(\underline{\mathbf{X}}, \Gamma))$ is a reducible tropical hypersurface; i.e. it can be expressed as the union of two proper tropical hypersurfaces. So, if we prove that for a special valuation $\mathbf{v}, \operatorname{Trop}(V(\underline{\mathbf{X}}, \Gamma))$ is irreducible, then $V(\underline{\mathbf{X}}, \Gamma)$ will be irreducible over any field $k$.

Theorem 1.6. Let $N \geq 3$. Given any field $K \supseteq k\left(A_{1}, \ldots, A_{N-1}\right)$, there exists a valuation $v$ defined over $K$ such that $\operatorname{Trop}(V(\underline{\mathbf{X}}, \Gamma))$ is an irreducible tropical variety if and only if:

- $\operatorname{dim}\left(\mathcal{L}_{\Gamma}\right) \geq 2$,

- $\operatorname{gcd}\left(\mathbf{X}^{\gamma_{i}}\right)_{1 \leq i \leq N}=1$ or equivalently $\bar{\gamma}=(0, \ldots, 0)$,

- $d_{\Gamma}=1$.

This result is optimal in the following sense: it is known that $\operatorname{Trop}(\{f=0\})$ does not depend on the field but only on the values $v\left(A_{i}\right)$.

Take for instance $f=\sum_{i=0}^{N} A_{i} \mathbf{Y}^{i}$ over a field of characteristic zero with $d_{\Gamma}>1$, and let $g:=\sum_{i=0}^{N} B_{i} \mathbf{Y}^{i}$ be a polynomial with the same support and with coefficients in a field of characteristic $p \mid d_{\Gamma}$. Give to these polynomials valuations $v$ and $v^{\prime}$ such that $v^{\prime}\left(B_{i}\right)=v\left(A_{i}\right)$. In these conditions, we will have

$$
\operatorname{Trop}(\{g=0\})=\operatorname{Trop}(\{f=0\}),
$$


but $g=\left(\sum_{i=0}^{N} B_{i}^{1 / p} \mathbf{Y}^{i / p}\right)^{p}$ factorizes in the algebraic closure of its field of definition; hence $\operatorname{Trop}(\{f=0\})$ will always be reducible. So, the tropical criteria will not help to deduce the irreducibility of $f$.

The paper is organized as follows: in Section 2, we give explicit conditions for the irreducibility of the Vandermonde variety. In Section 3 we prove Theorem 1.5 . We conclude by introducing some tools from tropical geometry and by proving Theorem 1.6 in Section 4 .

\section{BERTINI'S THEOREM AND THE IRREDUCIBILITY OF THE VANDERMONDE VARIETY}

We begin by studying the geometric irreducibility of the variety defined by $V(\underline{\mathbf{X}}, \Gamma)$ in $\bar{k}^{N n}$. In order to do this, we will apply one of the several versions of Bertini's theorem given in [Jou]. Recall the following ([Jou, Definition 4.1]):

Definition 2.1. A $k$-scheme $\mathcal{V}$ over a field $k$ is said to be geometrically irreducible if $\mathcal{V} \otimes_{k} \bar{k}$ is an irreducible scheme.

Now we are ready to present the version of Bertini's theorem that we will use.

Theorem 2.2 ([Jou, Théorème 6.3). Let $k$ be an infinite field, $\mathcal{V}$ a geometrically irreducible $k$-scheme of finite type, $\mathbb{E}_{k}^{m}$ the affine space of dimension $m$, and $f: \mathcal{V} \rightarrow \mathbb{E}_{k}^{m}$ ak-morphism, i.e.

$$
\begin{array}{rlcc}
f: \mathcal{V} & \rightarrow & \mathbb{E}_{k}^{m} \\
z & \mapsto & \left(f_{1}(z), \ldots, f_{m}(z)\right)
\end{array}
$$

with $f_{i} \in \Gamma\left(\mathcal{V}, \mathcal{O}_{\mathcal{V}}\right)$. If $\mathcal{V}$ is geometrically irreducible and $\operatorname{dim}(\overline{f(\mathcal{V})}) \geq 2$, then for almost all $\xi \in k^{m+1}, f^{-1}\left(\left\{z \in \mathbb{E}_{k}^{m}: \xi_{0}+\xi_{1} z_{1}+\ldots+\xi_{n} z_{n}=0\right\}\right)$ is geometrically irreducible.

Definition 2.3. Let $\Gamma=\left\{\gamma_{1}, \ldots, \gamma_{N}\right\} \subset \mathbb{N}^{n}, \mathbf{Y}:=\left(Y_{1}, \ldots, Y_{n}\right)$ be a set of $n$ variables and $A_{i}, 1 \leq i \leq N$, be another set of indeterminates. The generic polynomial supported in $\Gamma$ is defined as

$$
P(\mathbf{Y}, \Gamma):=\sum_{i=1}^{N} A_{i} \mathbf{Y}^{\gamma_{i}}
$$

Proposition 2.4. If $\operatorname{dim}\left(\mathcal{L}_{\Gamma}\right) \geq 2$ and $\operatorname{gcd}\left(\mathbf{X}^{\gamma_{i}}\right)_{1 \leq i \leq N}=1$, then $P(\mathbf{Y}, \Gamma)$ defines an irreducible set in ${\overline{k\left(A_{1}, \ldots, A_{N}\right)}}^{n}$.

Proof. Note that $\operatorname{dim}\left(\mathcal{L}_{\Gamma}\right) \geq 2$ implies $N \geq 3$ and, moreover, that there are three components of $\Gamma$ that are not collinear. We pick a triple of vectors with this property which we suppose w.l.o.g. are $\gamma_{1}, \gamma_{2}, \gamma_{3}$.

In order to use Theorem 2.2, let $\mathcal{V}:=\operatorname{Spec}\left(k\left[Y_{1}^{ \pm 1}, \ldots, Y_{n}^{ \pm 1}\right]\right)$ be the torus $\left(k^{*}\right)^{n}$, and set

$$
\begin{aligned}
& f: \mathcal{V} \rightarrow \quad \mathbb{E}_{k}^{N-1} \\
& z \mapsto \quad\left(z^{\gamma_{2}-\gamma_{1}}, z^{\gamma_{3}-\gamma_{1}}, \ldots, z^{\gamma_{N}-\gamma_{1}}\right) .
\end{aligned}
$$


By hypothesis, the rank of the matrix $\left(\begin{array}{c}\gamma_{2}-\gamma_{1} \\ \gamma_{3}-\gamma_{1} \\ \ldots \\ \gamma_{N}-\gamma_{1}\end{array}\right)$ is at least two. So, the top two-by-two submatrix of its Smith normal form is $\left|\begin{array}{cc}d_{1} & 0 \\ 0 & d_{2}\end{array}\right|=d_{1} d_{2} \neq 0$. Hence it follows that under a suitable monomial change of coordinates, the map $f$ is of the form $z \mapsto\left(z_{1}^{d_{1}}, z_{2}^{d_{2}}, \ldots\right)$; this shows that the dimension of the image of $f$ is greater than one, so we can apply Bertini's theorem and have that for almost all $\xi \in \bar{k}^{N}$, the polynomial $Q(\xi, \mathbf{Y}):=\sum_{i=1}^{N} \xi_{i} \mathbf{Y}^{\gamma_{i}}$ defines an irreducible set in $\left(\bar{k}^{*}\right)^{n}$. The fact that $\operatorname{gcd}\left(\mathbf{Y}^{\gamma_{i}}\right)_{1 \leq i \leq N}=1$ implies that $Q(\xi, \mathbf{Y})$ defines an irreducible set also in $\bar{k}^{n}$ for almost all $\xi$, and hence that the claim holds for $P(\mathbf{Y}, \Gamma)$.

Proposition 2.5. Let $\Gamma=\left(\gamma_{1}, \ldots, \gamma_{N}\right) \subset \mathbb{N}^{n}$ with $N \geq 3$ and suppose that $\operatorname{gcd}\left(\mathbf{Y}^{\gamma_{i}}\right)_{1 \leq i \leq N}=1$. Then the following hold:

- If $\operatorname{dim}\left(\mathcal{L}_{\Gamma}\right)=1$, then $P(\mathbf{Y}, \Gamma)$ factorizes in $\overline{k\left(A_{1}, \ldots, A_{N}\right)}[\mathbf{Y}]$.

- If $\operatorname{dim}\left(\mathcal{L}_{\Gamma}\right)>1$, then

- if char $(k)$ does not divide $d_{\Gamma}$, then $P(\mathbf{Y}, \Gamma)$ is absolutely irreducible;

- if $\operatorname{char}(k)=p \mid d_{\Gamma}$, then $P(\mathbf{Y}, \Gamma)=R(\mathbf{Y})^{p^{r}}$, with $p^{r} \mid d_{\Gamma}, p^{r+1}$ not dividing $d_{\Gamma}$, and $R(\mathbf{Y}) \in \overline{k\left(A_{1}, \ldots, A_{N}\right)}[\mathbf{Y}]$ irreducible of support $\frac{1}{p^{r}} \Gamma$.

Proof. If the vertices are contained in an affine line, then, via a monomial transformation, we can reduce $P(\mathbf{Y}, \Gamma)$ to a univariate polynomial, which always factorizes (due to the fact that $N>2$ ) as a product of linear factors with coefficients in $\overline{k\left(A_{1}, \ldots, A_{N}\right)}$. The variety defined by this polynomial may be reducible or not, depending on the inseparability of this polynomial.

Suppose now that $\mathcal{L}_{\Gamma}$ has affine dimension at least two. Then we can apply the previous proposition and conclude that the variety defined by $P(\mathbf{Y}, \Gamma)$ is irreducible over $\overline{k\left(A_{1}, \ldots, A_{N}\right)}$.

Hence, there exist an irreducible polynomial $R(\mathbf{Y}) \in \overline{k\left(A_{1}, \ldots, A_{N}\right)}[\mathbf{Y}]$ and a $D \in \mathbb{N}$ such that

$$
P(\mathbf{Y}, \Gamma)=R(\mathbf{Y})^{D}
$$

It is clear that $R(\mathbf{Y})$ cannot be a monomial. If $D=1$, then we are done. Suppose then that $D>1$ and that $p:=\operatorname{char}(k)$ is coprime with $d_{\Gamma}$. We can suppose w.l.o.g. that $p$ does not divide the first coordinate of one of the $\gamma_{i}$ 's. But then we have

$$
0 \neq \frac{\partial P(\mathbf{Y}, \Gamma)}{\partial \mathbf{Y}_{1}}=\sum_{i=1}^{N} \gamma_{i 1} A_{i} \mathbf{Y}^{\gamma_{i}-\mathbf{e}_{1}}=D R(\mathbf{Y})^{D-1} \frac{\partial R(\mathbf{Y})}{\partial \mathbf{Y}_{1}}
$$

In particular, we get that $p$ does not divide $D$. As $R(\mathbf{Y})$ is not a monomial, it turns out that $\frac{\partial P(\mathbf{Y}, \Gamma)}{\partial \mathbf{Y}_{1}}$ has at least two nonzero terms. This implies that $P(\mathbf{Y}, \Gamma)$ has at least two different monomials with positive degree on $\mathbf{Y}_{1}$, so the degree of $R(\mathbf{Y})$ with respect to the first variable is positive and, due to (1), the same applies to $\frac{\partial P(\mathbf{Y}, \Gamma)}{\partial \mathbf{Y}_{1}}$.

We can then eliminate $\mathbf{Y}_{1}$ by computing the univariate (or classical) resultant (see [GKZ] ) of the polynomials $P(\mathbf{Y}, \Gamma)$ and $\frac{\partial P(\mathbf{Y}, \Gamma)}{\partial \mathbf{Y}_{1}}$ with respect to the first variable. This is a polynomial in $k\left[A_{1}, \ldots, A_{n}, \mathbf{Y}_{2}, \ldots, \mathbf{Y}_{n}\right]$ which must be identically 
zero, as $R(\mathbf{Y})$ is a common factor of both $P(\mathbf{Y}, \Gamma)$ and $\frac{\partial P(\mathbf{Y}, \Gamma)}{\partial \mathbf{Y}_{1}}$. This is a contradiction to the fact that $A_{1}, \ldots, A_{n}, \mathbf{Y}_{2}, \ldots, \mathbf{Y}_{n}$ are algebraically independent.

Suppose now that $p:=\operatorname{char}(k) \mid d_{\Gamma}$, and let $r$ be the maximum integer such that $p^{r} \mid D$, so that we can write $D=p^{r} q$ with $(p, q)=1$. Write $R(\mathbf{Y})=\sum_{j=1}^{M} R_{j} \mathbf{Y}^{\gamma_{j}^{\prime}}$. We then have

$$
P(\mathbf{Y}, \Gamma)=\sum_{i=1}^{N} A_{i} \mathbf{Y}^{\gamma_{i}}=\left(\sum_{j=1}^{M} R_{j} \mathbf{Y}^{\gamma_{j}^{\prime}}\right)^{p^{r} q}=\left(\sum_{j=1}^{M} R_{j} p^{r} \mathbf{Y}^{p^{r} \gamma_{j}^{\prime}}\right)^{q} .
$$

From here, we deduce that $p^{r}$ divides $d_{\Gamma}$. Moreover, after dividing all the exponents by $p^{r}$ we get

$$
P_{r}(\mathbf{Y}, \Gamma):=\sum_{i=1}^{N} A_{i} \mathbf{Y}^{\frac{\gamma_{i}}{p^{r}}}=\left(\sum_{j=1}^{M} R_{j} p^{r} \mathbf{Y}^{\gamma_{j}^{\prime}}\right)^{q}
$$

It turns out that $P_{r}(\mathbf{Y}, \Gamma)=P\left(\mathbf{Y}, \frac{1}{p^{r}} \Gamma\right)$. An argument like the one above over $\frac{1}{p^{r}} \Gamma$ shows that $q$ cannot be different from one if the $A_{i}$ are algebraically independent. This completes the proof.

As in the introduction, for $\ell=1, \ldots, N$ we set

$$
\Delta_{\ell}(\underline{\mathbf{X}}, \Gamma):=\operatorname{det}\left(\mathbf{X}_{i}^{\gamma_{j}}\right) \underset{\substack{2 \\ j \neq i}}{\substack{j \\ j}, 1 \leq j \leq N}
$$

i.e. $\Delta_{\ell}$ is the minor obtained by deleting the first row and $\ell$-th column in the generalized Vandermonde matrix.

Theorem 2.6. For any index $\ell_{0}$, the family $\left\{\Delta_{\ell} / \Delta_{\ell_{0}}: \ell=1, \ldots N, \ell \neq \ell_{0}\right\}$ is algebraically independent over any field $k$.

Proof. Suppose without loss of generality that $\ell_{0}=N$. It is easy to see that $\Delta_{N}$ does not define the zero function on $\bar{k}^{(n-1) N}$ even if $\operatorname{char}(k)>0$.

Let $\mathcal{V}$ be the Zariski image of the rational map

$$
\begin{array}{ccc}
\bar{k}^{n(N-1)} & \longrightarrow \\
\left(\mathbf{X}_{2}, \ldots, \mathbf{X}_{N}\right) & \mapsto \quad\left(\mathbf{X}_{2}, \ldots, \mathbf{X}_{N}, \frac{\Delta_{1}}{\Delta_{N}}, \ldots, \frac{\Delta_{N-1}}{\Delta_{N}}\right)
\end{array}
$$

It is clear that this is a birational map between $\bar{k}^{n(N-1)}$ and $\mathcal{V}$. Let $\mathcal{I}$ be the ideal of $\mathcal{V}$ in $k\left[\mathbf{X}_{2}, \ldots, \mathbf{X}_{N}, a_{1}, \ldots, a_{N-1}\right] . \mathcal{I}$ is a prime ideal that contains the polynomials $\Delta_{i}-a_{i} \Delta_{N}, i<N$, and by Cramer's rule $f\left(\mathbf{X}_{\ell}\right)=\mathbf{X}_{\ell}^{\gamma_{N}}+\sum_{i=1}^{N-1} a_{i} \mathbf{X}_{\ell}^{\gamma_{i}}, 2 \leq \ell \leq N$. Let $\mathbf{a}=\left\{a_{1}, \ldots, a_{N-1}\right\}$. By construction, the field of rational functions of $\mathcal{V}$ is isomorphic to the field of fractions of the integer domain,

$$
\mathbb{L}=\operatorname{Frac}\left(\frac{k\left[\mathbf{X}_{2}, \ldots, \mathbf{X}_{N}, \mathbf{a}\right]}{\mathcal{I}}\right) \simeq k\left(\mathbf{X}_{2}, \ldots, \mathbf{X}_{N}\right) .
$$

In particular, $\left(\mathbf{X}_{2}, \ldots, \mathbf{X}_{N}\right)$ is a transcendence basis of $k \subset \mathbb{L}$ and the dimension of $\mathbb{L}$ is $n(N-1)$. For each index $2 \leq \ell \leq N$, choose one variable $X_{\ell, j_{\ell}}$ appearing in $f\left(\mathbf{X}_{l}\right)$. Denote by $\mathbf{X}_{0}=\left\{\mathbf{X}_{2}, \ldots, \mathbf{X}_{N}\right\} \backslash\left\{X_{2, j_{2}}, \ldots, X_{N, j_{N}}\right\}$ the remaining variables $X_{i, j}$ not chosen. As an element in $\mathbb{L}, X_{\ell, j_{\ell}}$ is algebraic over $k\left(\mathbf{X}_{0}, \mathbf{a}\right)$, because $f\left(X_{\ell, j_{\ell}}\right) \in \mathcal{I}$. So $\mathbb{L}$ itself is an algebraic extension of $k\left(\mathbf{X}_{0}, \mathbf{a}\right)$. The set $\left\{\mathbf{X}_{0}, \mathbf{a}\right\}$ is of cardinality $(n-1)(N-1)+(N-1)=n(N-1)$. So it is a transcendence basis of $\mathbb{L}$ over $k$. In particular, this means that the set $\left\{a_{1}, \ldots a_{N-1}\right\}$ is algebraically 
independent over $\mathbb{L}$, and hence $\left\{\frac{\Delta_{1}}{\Delta_{N}}, \ldots, \frac{\Delta_{N-1}}{\Delta_{N}}\right\}$ is algebraically independent over $k$.

\section{Proof of Theorem 1.5}

With all the preliminaries given in Section 2, we can prove the main theorem. It is clear that if any of the three conditions in the statement of Theorem 1.5 fail to hold, then $V(\underline{\mathbf{X}}, \Gamma)$ factorizes.

Suppose then that these conditions are satisfied. By expanding $V(\underline{\mathbf{X}}, \Gamma)$ as a polynomial in the variables indexed by $\mathbf{X}_{1}$, we have

$$
V(\underline{\mathbf{X}}, \Gamma)=\sum_{i=1}^{N}(-1)^{\sigma_{i}} \Delta_{i} \mathbf{X}_{1}^{\gamma_{i}}
$$

with $\sigma_{i} \in\{0,1\}$. Hence, we can regard $V(\underline{\mathbf{X}}, \Gamma)$ as the polynomial $P\left(\mathbf{X}_{1}, \Gamma\right)$ specialized under $A_{i} \mapsto(-1)^{\sigma_{i}} \Delta_{i}$.

As the family $\left((-1)^{\sigma_{i}} \Delta_{i} / \Delta_{N}\right)_{1 \leq i \leq N-1}$ is algebraically independent (due to Theorem [2.6), the polynomial $\mathbf{X}_{1}^{\gamma_{N}}+\sum_{i=1}^{N-1}(-1)^{\sigma_{i}} \Delta_{i} / \Delta_{N} \mathbf{X}_{1}^{\gamma_{i}}$ is generic among the polynomials of support $\Gamma$ that are monic in $\gamma_{1}$. This means that for almost every $t_{i j}$ with $2 \leq i \leq N$ and $1 \leq j \leq N$, the set of zeroes of $V(\underline{\mathbf{X}}, \Gamma)$ in $\bar{k}^{n}$ after setting $X_{i j} \mapsto t_{i j}$ is irreducible (by Proposition 2.4).

As a consequence of this, we get that the set of zeroes of $V(\underline{\mathbf{X}}, \Gamma)$ is irreducible in $\overline{k\left(\mathbf{X}_{2}, \ldots, \mathbf{X}_{N}\right)}$ and hence, as in Proposition 2.5, that $V(\underline{\mathbf{X}}, \Gamma)$ must be the power of an irreducible polynomial. By again using Proposition 2.5 and our hypothesis, we conclude that $V(\underline{\mathbf{X}}, \Gamma)$ is irreducible in $\overline{k\left(\mathbf{X}_{2}, \ldots, \mathbf{X}_{N}\right)}\left[X_{1}\right]$.

In order to show irreducibility in $\bar{k}\left[\mathbf{X}_{1}, \mathbf{X}_{2}, \ldots, \mathbf{X}_{N}\right]$, we argue as follows: if $V(\underline{\mathbf{X}}, \Gamma)$ does factorize in this ring, then it must have an irreducible factor depending only on $\mathbf{X}_{2}, \ldots, \mathbf{X}_{N}$. It cannot be a monomial by the second hypothesis. So, it is a proper factor of positive degree in (we can assume w.l.o.g.) $\mathbf{X}_{2}$ and degree zero in $\mathbf{X}_{1}$. We then have

$$
V(\underline{\mathbf{X}}, \Gamma)=p\left(\mathbf{X}_{2}, \ldots, \mathbf{X}_{N}\right) q\left(\mathbf{X}_{1}, \mathbf{X}_{2}, \ldots, \mathbf{X}_{N}\right)
$$

with $\operatorname{deg}_{\mathbf{X}_{2}}(p)>0$. By making the change of coordinates $\mathbf{X}_{2} \leftrightarrow \mathbf{X}_{1}$, we get

$$
-V(\underline{\mathbf{X}}, \Gamma)=p\left(\mathbf{X}_{1}, \mathbf{X}_{3}, \ldots, \mathbf{X}_{N}\right) q\left(\mathbf{X}_{2}, \mathbf{X}_{1}, \ldots, \mathbf{X}_{N}\right)
$$

If $\operatorname{deg}_{\mathbf{X}_{2}}\left(q\left(\mathbf{X}_{1}, \mathbf{X}_{2}, \ldots, \mathbf{X}_{N}\right)\right)>0$, then the negative of the right hand side of (2) is a factorization of $V(\underline{\mathbf{X}}, \Gamma)$ with two factors of positive degree in $\mathbf{X}_{1}$, a contradiction to the irreducibility over $\overline{k\left(\mathbf{X}_{2}, \ldots, \mathbf{X}_{N}\right)}\left[\mathbf{X}_{1}\right]$. So, we must actually have

$$
V(\underline{\mathbf{X}}, \Gamma)=p\left(\mathbf{X}_{2}, \ldots, \mathbf{X}_{N}\right) q\left(\mathbf{X}_{1}, \mathbf{X}_{3}, \ldots, \mathbf{X}_{N}\right)
$$

But now, if we set $\mathbf{X}_{1}=\mathbf{X}_{2}$ in $V(\underline{\mathbf{X}}, \Gamma)$, we get

$$
0=p\left(\mathbf{X}_{1}, \ldots, \mathbf{X}_{N}\right) q\left(\mathbf{X}_{2}, \mathbf{X}_{3}, \ldots, \mathbf{X}_{N}\right)
$$

a contradiction to the fact that neither $p$ nor $q$ is zero. Hence, the irreducibility of $V(\underline{\mathbf{X}}, \Gamma)$ follows. 


\section{The tropical APPROACH}

As explained in the introduction, the expression $V(\underline{\mathbf{X}}, \Gamma)=\sum_{i=1}^{N}(-1)^{\sigma_{i}} \Delta_{i} \mathbf{X}_{1}^{\gamma_{i}}$ corresponds to the expansion of the generalized Vandermonde determinant with respect to the first row of its defining matrix. We again dehomogenize this polynomial as

$$
\mathbf{X}_{1}^{\gamma_{N}}+\sum_{i=1}^{N-1} A_{i} \mathbf{X}_{1}^{\gamma_{i}}
$$

where the $A_{i}$ 's are algebraically independent over $k$ by Theorem 2.6 .

Given any rank one valuation $v: \overline{k\left(A_{1}, \ldots, A_{N-1}\right)} \rightarrow \mathbb{R}$, we define $\operatorname{Trop}(V(\underline{\mathbf{X}}, \Gamma))$, the tropicalization of $V(\underline{\mathbf{X}}, \Gamma)$, as the closure of $\{V(\underline{\mathbf{X}}, \Gamma)=0\} \subset \overline{k\left(A_{1}, \ldots, A_{N-1}\right)} n$ under this valuation.

Let $\Lambda \subset \mathbb{R}^{n}$ be the convex hull of $\Gamma$. The values $v\left(A_{i}\right)$ define a regular subdivision $\operatorname{Subdiv}(\Lambda)$ that is combinatorially dual to $\operatorname{Trop}(V(\underline{\mathbf{X}}, \Gamma))([\mathrm{Mik}$, Proposition 3.11]). In particular, by the duality, the vertices of $\operatorname{Subdiv}(\Lambda)$ correspond to the connected components of $\mathbb{R}^{n} \backslash \operatorname{Trop}(V(\underline{\mathbf{X}}, \Gamma))$, the edges of $\operatorname{Subdiv}(\Lambda)$ correspond to the facets of $\operatorname{Trop}(V(\underline{\mathbf{X}}, \Gamma))$ and the two-dimensional polytopes of $\operatorname{Subdiv}(\Lambda)$ correspond to the ridges of $\operatorname{Trop}(V(\underline{\mathbf{X}}, \Gamma))$. There are more cells, but we will focus only on these.

Every facet $F$ of $\operatorname{Trop}(V(\underline{\mathbf{X}}, \Gamma))$ has a multiplicity associated with it as follows: let $e$ be the corresponding dual edge of $F$ in $\operatorname{Subdiv}(\Lambda)$. The multiplicity of $F$ is defined to be $\#\left(e \cap \mathbb{Z}^{n}\right)-1$, the integer length of $e$. With this definition, the balancing condition on the ridges of $\operatorname{Trop}(V(\underline{\mathbf{X}}, \Gamma))$ holds: given any such ridge $R$, let $F_{1}, \ldots, F_{r}$ be the facets containing $R$ in their boundary, $m_{i}$ the multiplicity of $F_{i}$ and $v_{i}$ the primitive integer normal vector to the affine hyperplane generated by $F_{i}$ chosen with a compatible orientation. Then

$$
\sum_{i=1}^{r} m_{i} v_{i}=0
$$

We refer to Mik] or [TS] for more background on this subject. We will use the balancing condition to show the irreducibility of $\operatorname{Trop}(V(\underline{\mathbf{X}}, \Gamma))$.

Proof of Theorem 1.6. If one of the hypotheses of Theorem 1.6 is not fulfilled, then it is easy to find a field $k$ where $V(\underline{\mathbf{X}}, \Gamma)$ factors, and hence $\operatorname{Trop}(V(\underline{\mathbf{X}}, \Gamma))$ would be reducible. Suppose then that the three conditions hold and let $k$ be any field.

Consider $\mathbf{X}_{1}^{\gamma_{N}}+\sum_{i=1}^{N-1} A_{i} \mathbf{X}_{1}^{\gamma_{i}}$, where $A_{i}$ are rational functions in $\left\{\mathbf{X}_{2}, \ldots, \mathbf{X}_{N}\right\}$, algebraically independent over $k$. Hence, any function $v:\left\{A_{1}, \ldots, A_{N-1}\right\} \rightarrow \mathbb{R}$ can be extended to a valuation

$$
\mathbf{v}: \overline{k\left(\mathbf{X}_{2}, \ldots, \mathbf{X}_{N}\right)} \rightarrow \mathbb{R}
$$

For our proof, we need a function that induces a regular triangulation of $\Gamma$. We may take, for instance, any appropriate generic infinitesimal perturbation of the standard paraboloid lifting function

$$
v\left(A_{i}\right)=\sum_{j=1}^{n}\left(\left(\gamma_{i j}+\epsilon_{i j}\right)^{2}-\gamma_{N j}^{2}\right) .
$$

This function induces a Delaunay triangulation of the set of exponents $\left\{\gamma_{1}, \ldots, \gamma_{N}\right\}$ (see [GR]).

The tropicalization of $V(\underline{\mathbf{X}}, \Gamma)$ under this valuation is combinatorially dual to this triangulation. So, in particular, the ridges of $\operatorname{Trop}(V(\underline{\mathbf{X}}, \Gamma))$ are always intersections 
of three facets, because the dual cell of each is always a triangle. Moreover, for any such intersection, the compatible primitive vectors involved in the balancing condition are pairwise linearly independent.

Suppose that $\operatorname{Trop}(V(\underline{\mathbf{X}}, \Gamma))=H_{1} \cup H_{2}$. Let $F_{1}$ be a facet of $\operatorname{Trop}(V(\underline{\mathbf{X}}, \Gamma))$ and suppose that $F_{1} \subseteq H_{1}$. We want to prove that $\operatorname{Trop}(V(\underline{\mathbf{X}}, \Gamma)) \subseteq H_{1}$ as sets of points. Let $R$ be any ridge incident to $F_{1}$, and let $F_{2}$ and $F_{3}$ be the other two facets incident to $R$. Let $m_{i}$ be the weight of $F_{i}$ as a facet of $H_{1}$, so $m_{i}=0$ if and only if $F_{i}$ is not a facet of $H_{1}$. Let $v_{i}$ be the compatible primitive vector associated to $F_{i}$ and $R$. Since $F_{1} \in H_{1}$, its weight must be a positive integer, $m_{1}>0$.

From the balancing condition, we have that $m_{1} v_{1}+m_{2} v_{2}+m_{3} v_{3}=0$. Since $m_{1}>0$ and $v_{1}, v_{2}, v_{3}$ are pairwise linearly independent vectors, it must happen that $m_{2}>0$ and $m_{3}>0$; that is, $F_{2}$ and $F_{3}$ have positive weights, so they belong to $H_{1}$ as sets of points. To sum up, for any facet $F$ of $\operatorname{Trop}(V(\underline{\mathbf{X}}, \Gamma))$ belonging to $H_{1}$ it happens that the facets that are ridge-connected to $F$ also belong to $H_{1}$. Now, since $\Gamma$ is not contained in a line, it is known that $\operatorname{Trop}(V(\underline{\mathbf{X}}, \Gamma))$ is ridge-connected, that is, every two facets can be connected by a path of facets such that any two of them that are consecutive have a common ridge.

We can then conclude by induction, upon showing that $\operatorname{Trop}(V(\underline{\mathbf{X}}, \Gamma))=H_{1}$ as subsets of $\mathbb{R}^{n}$. In particular, $\operatorname{Trop}(V(\underline{\mathbf{X}}, \Gamma))$ cannot factorize as the union of two different tropical hypersurfaces, set-theoretically.

However, it could still happen that $\operatorname{Trop}(V(\underline{\mathbf{X}}, \Gamma))=H_{1} \cup H_{2}$ with $H_{1}=H_{2}$ as sets but differing only in the multiplicities of the facets. In that case, let $m_{i}^{1}$ and $m_{i}^{2}$ be the multiplicities of $F_{i}$ as a facet of $H_{1}$ and $H_{2}$ respectively. Then, it is easy to check that $m_{i}^{1} / m_{i}^{2}=p / q$ is a rational constant that does not depend on the facet. Thus, there are positive integers $m_{i}^{0}$ such that $m_{i}^{1}=k_{1} m_{i}^{0}$ and $m_{i}^{2}=k_{2} m_{i}^{0}$, where $k_{1}, k_{2} \in \mathbb{Z}_{>0}$ are constants not depending on the facet $i$. Hence, the multiplicity of $F_{i}$ as a facet of $H$ is $\left(k_{1}+k_{2}\right) m_{i}^{0}$, and this implies that every facet has a multiplicity which is a multiple of $k_{1}+k_{2} \geq 2$. By duality, every edge of $\operatorname{Subdiv}(\Lambda)$ will have length that is an integer multiple of $\left(k_{1}+k_{2}\right)$. It follows that $d_{\Gamma}$ is a multiple of $k_{1}+k_{2}$, which contradicts the hypotheses.

\section{ACKNOWLEDGMENTS}

The authors would like to thank David Cox for posing the problem of the irreducibility of Vandermonde determinants, J. I. Burgos and J. C. Naranjo for useful discussions, and the anonymous referee for helpful suggestions on how to improve the presentation of this work.

\section{REFERENCES}

[BP] Beelen, Peter; Pellikaan, Ruud. The Newton polygon of plane curves with many rational points. Special issue dedicated to Dr. Jaap Seidel on the occasion of his 80th birthday (Oisterwijk, 1999). Des. Codes Cryptogr. 21 (2000), no. 1-3, 41-67. MR.1801161 (2003c:14024)

[BG] Bieri, Robert; Groves, J. R. J. The geometry of the set of characters induced by valuations. J. Reine Angew. Math. 347 (1984), 168-195. MR733052 (86c:14001)

[CL] Chui, Charles K.; Lai, Hang-Chin. Vandermonde determinant and Lagrange interpolation in $R^{s}$. Nonlinear and convex analysis (Santa Barbara, Calif., 1985), 23-35, Lecture Notes in Pure and Appl. Math., 107. Dekker, New York, 1987. MR892779 (88j:41011)

[EKL] Einsiedler, Manfred; Kapranov, Mikhail; Lind, Douglas. Non-Archimedean amoebas and tropical varieties. J. Reine Angew. Math. 601 (2006), 139-157. MR2289207 (2007k:14038) 
[EIM] El-Mikkawy, Moawwad. Vandermonde interpolation using special associated matrices. Appl. Math. Comput. 141 (2003), no. 2-3, 589-595. MR.1972934(2004a:65011)

[Gao] Gao, Shuhong. Absolute irreducibility of polynomials via Newton polytopes. J. Algebra 237 (2001), no. 2, 501-520. MR1816701 (2002f:52013)

[GS] Gasca, Mariano; Sauer, Thomas. Polynomial interpolation in several variables. Adv. Comput. Math. 12 (2000), no. 4, 377-410. MR1768957|(2001d:41010)

[GKZ] Gel'fand, I. M.; Kapranov, M. M.; Zelevinsky, A. V. Discriminants, resultants, and multidimensional determinants. Mathematics: Theory \& Applications. Birkhäuser Boston, Inc., Boston, MA, 1994. MR1264417 (95e:14045)

[GV] Giosan, Traian; Voicu, M. Formules d'approximation et d'interpolation par polynômes obtenues à l'aide du déterminant Vandermonde. Bul. Inst. Politehn. Iaşi (N.S.) 16 (20) (1970), facs. 3-4, sect. III, 13-19. MR0282497(43:8208)

[GR] Goodman, Jacob E.; O'Rourke, Joseph (editors). Handbook of discrete and computational geometry. Discrete Mathematics and its Applications. Chapman \& Hall/CRC, Boca Raton, FL, second edition, 2004. MR2082993 (2005j:52001)

[Gow] Gow, R. Cauchy's matrix, the Vandermonde matrix and polynomial interpolation. Irish Math. Soc. Bull. 28 (1992), 45-52. MR1205443 (94i:15002)

[Jou] Jouanolou, Jean-Pierre. Théorèmes de Bertini et applications. Progress in Mathematics, 42. Birkhäuser Boston, Inc., Boston, MA, 1983. MR725671 (86b:13007)

[LS] Little, John; Schwarz, Ryan. On toric codes and multivariate Vandermonde matrices. Appl. Algebra Engrg. Comm. Comput. 18 (2007), no. 4, 349-367. MR2322944 (2008h:94117)

[Mac] Macdonald, I. G. Symmetric functions and orthogonal polynomials. Dean Jacqueline B. Lewis Memorial Lectures presented at Rutgers University, New Brunswick, NJ. University Lecture Series, 12. American Mathematical Society, Providence, RI, 1998. MR.1488699 (99f:05116)

[Mik] Mikhalkin, Grigory. Enumerative tropical algebraic geometry in $\mathbb{R}^{2}$. J. Amer. Math. Soc. 18 (2005), no. 2, 313-377. MR2137980 (2006b:14097)

[Olv] Olver, Peter J. On multivariate interpolation. Stud. Appl. Math. 116 (2006), no. 2, 201-240. MR 2200737 (2007b:41055)

[Ost] Ostrowski, A. M. On multiplication and factorization of polynomials. II. Irreducibility discussion. Aequationes Math. 14 (1976), no. 1/2, 1-31. MR0422229 (54:10220)

[Tab] Tabera, L. F. Two tools in algebraic geometry: Construction of configurations in tropical geometry and hypercircles for the simplification of parametric curves. Ph.D. Thesis, Universidad de Cantabria/Université de Rennes I, 2007.

[TS] Tevelev, Jenia; Sturmfels, Bernd. Elimination theory for tropical varieties. Math. Res. Lett. 15 (2008), no. 3, 543-562. MR.2407231

[Zhu] Zhu, Ping. Multivariate Vandermonde determinants and general Birkhoff interpolation. Northeast. Math. J. 21 (2005), no. 3, 336-344. MR2173614(2006d:41003)

Departament D’Àlgebra i Geometria, Universitat de Barcelona, Gran Via 585, E-08007 BARCELONA, Spain

E-mail address: cdandrea@ub.edu

URL: http://carlos.dandrea.name/

Departamento de Matemáticas, Universidad de Cantabria, Estadística y ComputaCión, Av. los Castros S/N, E-39005, Santander, Spain

$U R L:$ http://personales.unican.es/taberalf/

Current address: Department of Mathematics, University of California, Berkeley, 893 Evans Hall \#3840, Berkeley, California 94720-3840

E-mail address: tabera@berkeley.edu

$U R L:$ http://math.berkeley.edu/ tabera 